\title{
MODELO DE MENSURAÇÃo PARA ADOÇ̃̃o DE PRÁTICAS DE ECOINOVAÇÃO: ANÁLISE DOS ELEMENTOS DA CULTURA DE INOVAÇÃO
}

\author{
MODEL OF MEASUREMENT FOR THE ADOPTION OF ECO-INNOVATION \\ PRACTICES: ANALYSIS OF THE ELEMENTS OF THE INNOVATION CULTURE
}

\author{
Marize Helena da Rosa Vendler \\ Universidade Estadual do Centro-Oeste do Paraná - UNICENTRO \\ marize00@gmail.com \\ Marlete Beatriz Maçaneiro \\ Universidade Estadual do Centro-Oeste do Paraná - UNICENTRO \\ marlete.beatriz@yahoo.com.br
}

Submissão:21/11/2019

Aprovação:29/12/2021

\begin{abstract}
RESUMO
Este artigo teve por objetivo desenvolver um modelo de mensuração, por meio da definição de indicadores construídos a partir da análise de elementos culturais, que possam favorecer a adoção de práticas de ecoinovação na indústria da construção, como fonte de capacidade competitiva. Trata-se de uma pesquisa caracterizada como descritiva e exploratória, de abordagem qualitativa e técnicas análise de conteúdo e triangulação. Os resultados apontam que os elementos culturais estudados favorecem a adoção de práticas de ecoinovação, por meio de fontes do ambiente que direcionam o desenvolvimento das práticas ambientais e inovadoras, que possibilitam as empresas obterem capacidade competitiva, pela apresentação de atributos de redução dos impactos ambientais. Sob esta ótica, desenvolveu-se um roteiro diagnóstico de indicadores, que pode ser um mecanismo estratégico, uma vez que oferece um instrumento de avaliação, o qual proporciona um feedback sobre o que precisa ser desenvolvido para conceber uma cultura de inovação com enfoque mais ecoinovador.
\end{abstract}

Palavras-chave: Elementos da cultura de inovação. Práticas de ecoinovação. Capacidade competitiva.

\begin{abstract}
The objective of this article is to develop a measurement model, by means of the definition of indicators constructed from the analysis of the cultural elements, that may favor the adoption of eco-innovation practices in the construction industry, as a source of competitive capacity. It is a research characterized as descriptive and exploratory, qualitative approach and techniques content analysis and triangulation. The results show that the studied cultural elements favor the adoption of eco-innovation practices, through environmental sources that guide the development of environmental and innovative practices, which allow companies to obtain competitive capacity, through the presentation of attributes of reduction of environmental impacts. From this perspective, a diagnostic roadmap for indicators has been developed, which can be a strategic mechanism, since it provides an evaluation tool, which provides feedback on
\end{abstract}


what needs to be developed to design a more eco-innovative approach to innovation culture.

Keywords: Elements of the culture of innovation. Eco-innovation practices. Competitive capacity.

\section{INTRODUÇÃO}

As inovações são vistas como um tema estratégico em Administração e relevantes mecanismos para o desenvolvimento sustentável, que proporcionam vantagens competitivas (NARANJO-VALENCIA; JIMÉNEZ; SANZ-VALLE, 2012) e possibilitam criar produtos e processos de caráter ambientalmente responsável (MACHADO, 2004; NILL; KEMP, 2009). A questão ambiental atualmente é uma das temáticas mais relavantes, devido envolver aspectos relacionados às organizações, como os métodos de produção mais limpos e a demanda dos consumidores por produtos ambientalmente mais corretos (NAGANO; VICK; MADEIRA, 2017).

As organizações, nesse sentido, são orientadas para atender as perspectivas ambientais, por meio de práticas, que segundo Souza, Lucas e Torres (2011, p. 212), são entendidas "como atividades reais da organização, desde as cotidianas até as mais inovadoras, as quais representam as manifestações culturais da organização". Nessa linha, as práticas organizacionais podem ser constituídas pela cultura de inovação, desenvolvida com o intuito de obter a diminuição da degradação ambiental e, assim, propiciar a adoção de ecoinovações.

As ecoinovações são concebidas por Arundel e Kemp (2009) como um conceito que se refere a inovações desenvolvidas visando obter a diminuição da degradação ambiental, as quais podem ser constituídas para alcançar motivações econômicas e ambientais. Assim, as práticas de ecoinovação das empresas podem proporcionar benefícios referentes aos negócios de otimização de recursos, de redução de custos, de incremento de produtividade e de competitividade. Isso implica na necessidade de avaliar os resultados dessas práticas por meio da utilização de ferramentas de mensuração, como indicadores, que sejam aplicados à realidade organizacional.

Nesse contexto, este estudo tem como objetivo desenvolver um modelo de mensuração, por meio da definição de indicadores construídos a partir da análise dos elementos culturais, que possam favorecer a adoção de práticas de ecoinovação na indústria da construção, como fonte de capacidade competitiva. Esses indicadores podem demonstrar o consumo de recursos naturais e iniciativas inovativas de gerenciamento ambiental.

A escolha de realizar o estudo no setor da construção deve-se ao fato de compreender que investimentos nesse setor são estratégicos para qualquer país, considerando que o desenvolvimento dele acarreta em prover necessidades tidas como básicas para a sociedade, como moradia; usinas hidroelétricas; entre outras construções. Entretanto, segundo PachecoTorgal e Labrincha (2013), estima-se que o setor da construção consuma mais matérias-primas que os demais e que gera mais impactos sobre o meio ambiente. Isto ocasiona a necessidade de práticas ambientais, que podem conduzir a adoção de ecoinovações.

Diante dessas considerações, para que a adoção de ecoinovações se torne uma prática organizacional consolidada, necessita-se de cultura que propicie a adoção de inovações ambientais voltadas para alcançar capacidade competitiva. Isso implica em estudar indicadores da cultura de inovação que orientem a constituição de conhecimentos sobre práticas de ecoinovação e competitividade organizacional. 


\section{REVISÃO DE LITERATURA}

\subsection{CONCEITO E CARACTERÍSTICAS DOS INDICADORES AMBIENTAIS}

A adoção de postura ambiental é estimulada pelo atendimento de indicadores estabelecidos para diminuição da degradação do meio ambiente, visando o desenvolvimento sustentável. Os indicadores de sustentabilidade são ferramentas que têm como função principal monitorar e fornecer informações sobre as dimensões ambientais, econômicas, socioeconômicas, culturais, entre outras, que compõem o desenvolvimento sustentável (CARVALHO et al., 2011).

A partir da utilização de indicadores ambientais, deve ser possível analisar as condições e mudanças da qualidade ambiental, bem como servir como ferramenta de suporte no processo de tomada de decisão, formulação de políticas e práticas sustentáveis ambientais (GOMES; MALHEIROS, 2012). Isso, considerando que os indicadores "têm a capacidade de descrever um estado ou uma resposta dos fenômenos que ocorrem em um meio" (KEMERICH; RITTER; BORBA, 2014, p. 3724). Assim, os indicadores devem ser claros e de fácil compreensão, a fim de serem utilizados como instrumento de avaliação.

As organizações que atuam nos contextos sociais e ambientais, conseguem atender o desafio de manter a competitividade e sua postura socialmente responsável e assim atender as pressões de diferentes stakeholders (MELO et al., 2017). Essa perspectiva enfatiza a relevância dos indicadores na realização de estudos que buscam alternativas para a solução dos problemas ambientais advindos das atividades produtivas de setores que utilizam em excesso os recursos naturais, como é o caso do setor da construção. Dessa forma, nesses setores é essencial a constituição de um sistema construtivo que considere a variável ambiental na operacionalização das práticas organizacionais.

A adoção de práticas para atender os aspectos ambientais pode ser direcionada pela cultura, que por meio de seus elementos orientam o comportamento dos membros da organização. Essa orientação pode ser realizada a partir da constituição de indicadores referentes aos elementos culturais, que apresentem objetivos ambientais e inovativos. A cultura que impulsiona objetivos inovativos é denominada de cultura de inovação.

\subsection{CULTURA DE INOVAÇÃO}

A temática da cultura de inovação é relativamente recente na literatura (MEISSNER; SPRENGER, 2011). Esse tema surgiu da perspectiva defendida por pesquisadores que evidenciam a contribuição da cultura organizacional nos processos de inovação, o que constitui um fator estratégico para a organização (SCHREIBER et al., 2016). Dessa forma, a cultura organizacional, que incentiva a capacidade de inovação, que tolera risco e apoia o crescimento pessoal e o desenvolvimento, pode ser denominada como cultura de inovação (MARTÍN-DE CASTRO et al., 2013).

Nesse contexto, a cultura tem um papel crucial para o desenvolvimento da inovação (TURRO; URBANO; PERIS-ORTIZ, 2014), que se fundamenta na concepção de inovações, as quais necessitam de elementos que direcionem os comportamentos para propósitos inovadores. Para Freitas (1991), Schein (1984) e Machado (2004), os principais elementos da cultura, que podem ser desenvolvidos para atender o âmbito da inovação, são: valores; crenças e pressupostos; ritos, rituais e cerimônias; estórias e mitos; tabus; heróis; normas; comunicação; e artefatos e símbolos.

A identificação dos elementos que compõem a cultura de inovação é um dos principais objetivos dos pesquisadores, como o estudo de Dombrowski et al. (2007), que evidenciou oito elementos da cultura de inovação: 1) missão inovadora e declarações de visão;2) comunicação 
democrática lateral; 3) espaços seguros; 4) flexibilidade; 5) expansão de fronteiras; 6) colaboração; 7) incentivos; e 8) liderança. Com base nesses elementos, Janiunaite e Petraite (2010) demonstraram nas suas pesquisas que, dos oito elementos, três são essenciais para o surgimento de inovações nas organizações, sendo eles: a declaração de missão e visão (proporcionam as diretrizes para os empregados e para as práticas de trabalho); a comunicação democrática (comunicação sem barreiras na organização); e a colaboração (compartilhamento de informações entre funcionários e parceiros e nas diversas unidades da organização).

A partir da estrutura cultural de Schein (1984), composta por artefatos, valores compartilhados e pressupostos básicos, também os autores Hogan e Coote (2013) desenvolveram um modelo que identificou oito dimensões da cultura organizacional de apoio à inovação: 1) sucesso; 2) abertura e flexibilidade; 3) comunicação interna; 4) competência e profissionalismo; 5) cooperação interfuncional; 6) responsabilidade; 7) apreciação; e 8) assunção a risco. Através dessas dimensões, a organização incentiva os comportamentos para propósitos inovadores.

Dessa forma, pode-se inferir que, para compreender se a cultura de uma organização é apropriada ao desenvolvimento da inovação, é necessário entender os elementos desta. Percebese que a cultura se relaciona com a inovação, tendo em vista que pode estimular o comportamento inovador dos indivíduos. Ela pode conduzir os colaboradores a entender e a compartilhar a inovação como um valor fundamental da organização, tendo compromisso em promovê-la (NARANJO-VALENCIA; SANZ-VALLE; JIMENEZ-JIMENEZ，2010). A promoção da inovação pode ser desenvolvida buscando a eficiência em termos econômicos e ambientais, o que conduz a adoção de práticas de ecoinovação voltadas para alcançar a capacidade competitiva.

\subsection{PRÁTICAS DE ECOINOVAÇÃO COMO UMA FONTE DE CAPACIDADE COMPETITIVA}

Na organização, a gestão com enfoque ambiental é vista como uma possibilidade de as empresas se tornarem aliadas do desenvolvimento sustentável (THEIS; SCHREIBER, 2017). Também proporciona a obtenção de capacidade competitiva, por meio de processos produtivos que promovam a eficiência ambiental requerida pela sociedade e clientes. Essa capacidade competitiva, quando ligada a propósitos inovativos, conduz à adoção de ecoinovação.

A ecoinovação é um processo de mudança tecnológica e/ou social sistêmica, que consiste na invenção de uma ideia e sua aplicação na prática da melhoria do desempenho ambiental e econômico (KÖNNÖLÄ; CARRILLO-HERMOSILLA; GONZALEZ, 2008). Assim, as organizações que adotam práticas de ecoinovação apresentam concepções trazidas nos estudos de Schumpeter (1997), o qual ressalta que, para alcançar o desenvolvimento econômico, as empresas terão que incorporar mudanças como novas tecnologias e assim manter a posição competitiva no mercado.

Essa posição pode ser avaliada de acordo com a preferência dos consumidores pelos produtos que a empresa oferece. Nesse sentido, a "competitividade resulta da transformação de ideias em produtos de uma forma mais rápida, barata e qualitativamente melhores e mais apeteciveis que os concorrentes, aos olhos do mercado e dos consumidores finais" (DIAS, 2015 , p. 3). Isso pode implicar em utilizar menos recursos, como os naturais, o que torna os produtos mais baratos e conduz a redução dos impactos ambientais advindos dos processos produtivos.

Nesse contexto, para atender e conquistar diferencial competitivo, as organizações podem direcionar suas atenções para introduzir ecoinovações como alternativa que integra o desenvolvimento econômico e o ambiental, com a melhoria da competitividade (RENNINGS; 
RAMMER, 2011; MAÇANEIRO; CUNHA, 2014; GAZIULUSOY; BREZET, 2015). Dessa forma, a ecoinovação surge como um fator relevante para constituir estratégias e práticas que visem o desenvolvimento econômico e a conservação da competitividade.

Nessa conjuntura, torna-se primordial as organizações desenvolverem ecoinovações, para gerar novas oportunidades de investimentos, que fomentam o desenvolvimento econômico e proporcionam a capacidade competitividade. Essas oportunidades podem ser analisadas frente à ênfase em ecoinovação como um fator de competitividade. Isso é evidenciado por Ciasullo e Troisi (2013), ao considerarem que o processo de ecoinovação reflete positivamente nas práticas competitivas.

O investimento em questões ambientais pelas organizações, segundo Singh et al. (2014), tem como principal razão a possibilidade de melhoria do desempenho econômico. Para Fraj, Matute e Melero (2015), a inovação e a estratégia ambiental proativa beneficiam a competitividade organizacional. Dessa forma, as ecoinovações geram novas oportunidades de investimentos, que fomentam o desenvolvimento econômico e proporcionam a competitividade organizacional.

\section{METODOLOGIA}

A pesquisa deste estudo possui abordagem qualitativa, que procura trabalhar dados oriundos de representações dos atos e das expressões humanas (GODOI; BALSINI, 2010). Assim, buscou descrever e analisar os indicadores dos elementos da cultura de inovação e as práticas de ecoinovação, como uma fonte de capacidade competitiva, propondo um modelo de mensuração. Considerou-se também esta pesquisa como exploratória, devido à literatura não explorar especificamente as questões que relacionem os temas cultura de inovação e as práticas de ecoinovação.

Para compor o modelo, foi utilizada a estratégia de estudo de casos múltiplos, desenvolvida em cinco indústrias do setor da construção, localizadas nas regiões Centro-Oeste e Oeste do Paraná. As fontes de evidências contemplam entrevistas semiestruturadas, como fonte principal; observação; diário de pesquisa; e análise documental dos materiais informativos das empresas, dos tipos físicos ou online (site das empresas). As entrevistas foram realizadas com dois representantes de cada empresa, sendo um gestor, que na maioria dos casos foi o sócio-proprietário da empresa e um funcionário que atua na área ambiental, sendo que este, em todos os casos, é graduado em engenharia. O período de realização da coleta de dados foi de outubro a novembro de 2016 e o estudo como um todo se desenvolveu entre os anos de 2016 e 2017.

O tratamento, a análise e a interpretação das evidências foram realizados por meio das técnicas de triangulação, que busca relacionar os dados coletados por meio das diferentes fontes, com a revisão de literatura. Também foi utilizada a técnica de análise de conteúdo (BARDIN, 2004), através da análise temática, descrita por Bardin (2004). Essa análise possibilita a compreensão dos dados por meio do uso de unidades de análise temáticas, as quais são recortes do texto como sentenças, frases ou parágrafos, que reúnem unidades de registro, em razão das características comuns.

Dessa forma, a análise foi realizada através da transcrição dos relatos dos entrevistados, com a identificação de unidades de análise temática e do cruzamento entre as fontes de evidências e a teoria apresentada. Assim, foram definidas categorias de análise dos elementos da cultura de inovação, para definir indicadores a partir dos pontos de congruências de cada categoria. O Quadro 1 apresenta essas categorias com sua abrangência constitutiva e embasamento teórico. 
Quadro 1: Definições constitutivas das categorias de análise

\begin{tabular}{|c|c|c|}
\hline $\begin{array}{l}\text { Categorias de } \\
\text { Análise }\end{array}$ & Definições Constitutivas & $\begin{array}{l}\text { Embasamento (autores } \\
\text { representativos) }\end{array}$ \\
\hline $\begin{array}{l}\text { Adaptabilidade e } \\
\text { flexibilidade }\end{array}$ & $\begin{array}{l}\text { Adequação das questões culturais, por meio de uma } \\
\text { abordagem flexível, que possibilite promover ações } \\
\text { para compreender e atender as demandas de } \\
\text { preservação do meio ambiente. }\end{array}$ & $\begin{array}{l}\text { Dombrowski et al. (2007); } \\
\text { Janiunaite e Petraite (2010); } \\
\text { Hogan e Coote (2013). }\end{array}$ \\
\hline $\begin{array}{l}\text { Regulamentações e } \\
\text { políticas ambientais }\end{array}$ & $\begin{array}{l}\text { A legislação ambiental, estabelecida pelo governo; } \\
\text { setor de atuação da organização; normas operacionais e } \\
\text { práticas, bem como os compromissos da organização } \\
\text { que propiciam a diminuição da degradação ao meio } \\
\text { ambiente. }\end{array}$ & $\begin{array}{l}\text { Machado (2004); Rennings e } \\
\text { Rammer (2011); Farias et al. } \\
\text { (2012); Sanches e Gomes } \\
\text { (2015). }\end{array}$ \\
\hline $\begin{array}{l}\text { Orientação para } \\
\text { mercado }\end{array}$ & $\begin{array}{l}\text { A busca pela compreensão da dinâmica do mercado, } \\
\text { com o intuito de atualizar informações, bem como } \\
\text { desenvolver e acessar inovações tecnológicas para } \\
\text { atender aos objetivos ambientais da organização. }\end{array}$ & $\begin{array}{l}\text { Apekey et al. (2011); } \\
\text { Martins, Martins e } \\
\text { Terblanche (2004). }\end{array}$ \\
\hline Crenças e valores & $\begin{array}{l}\text { Os elementos aceitos pela organização como } \\
\text { importantes para o atendimento dos seus objetivos, que } \\
\text { direcionam os comportamentos para adoção de práticas } \\
\text { de ecoinovação dentro do âmbito organizacional. }\end{array}$ & $\begin{array}{l}\text { Schein (1984); Freitas } \\
\text { (1991); Machado (2004); } \\
\text { Apekey et al. }(2011)\end{array}$ \\
\hline Estrutura & $\begin{array}{l}\text { Consiste na estrutura organizacional, tanto tangível } \\
\text { (sistemas operacionais, símbolos etc), como } \\
\text { intangíveis, uma gestão que valoriza a autonomia, o } \\
\text { trabalho em equipe etc.), que orientam práticas no } \\
\text { sentido de conduzir o desenvolvimento de inovação em } \\
\text { concordância com os recursos ambientais. }\end{array}$ & $\begin{array}{l}\text { Martins e Terblanche } \\
\text { (2003); Dombrowski et al. } \\
\text { (2007); Saran, Servieri e } \\
\text { Kalliny (2009); Hogan e } \\
\text { Coote (2013). }\end{array}$ \\
\hline $\begin{array}{l}\text { Estímulo à } \\
\text { inovação }\end{array}$ & $\begin{array}{l}\text { Os aspectos presentes na cultura, que incentivam a } \\
\text { criatividade e a inovação, tais como o reconhecimento } \\
\text { de novas ideias, os quais possibilitam formalização de } \\
\text { inovações com perspetivas ambientais. }\end{array}$ & $\begin{array}{l}\text { Martins, Martins e } \\
\text { Terblanche (2004); Martín- } \\
\text { de Castro et al. (2013); } \\
\text { Hogan e Coote (2013). }\end{array}$ \\
\hline $\begin{array}{l}\text { Comunicação } \\
\text { aberta }\end{array}$ & $\begin{array}{l}\text { Fluxo de informações que proporciona a compreensão } \\
\text { clara quanto às mudanças e troca de ideias, para buscar } \\
\text { os melhores resultados referentes às questões } \\
\text { ambientais. }\end{array}$ & $\begin{array}{l}\text { Dombrowski et al. (2007); } \\
\text { Janiunaite e Petraite (2010); } \\
\text { Hogan e Coote (2013); } \\
\text { Sanches e Gomes (2015). }\end{array}$ \\
\hline
\end{tabular}

Fonte: elaboração própria.

Assim, a análise consistiu na codificação por unidades temáticas, reunindo os recortes de texto dos dados coletados, de acordo com o critério semântico, ou seja, os significados que a mensagem propicia.

\section{ANÁLISE DE DADOS, DISCUSSÕES E PROPOSIÇÕES}

\subsection{FONTES DO AMBIENTE QUE FAVORECEM A ADOÇÃO DE PRÁTICAS DE ECOINOVAÇÃO}

Inicialmente, buscou-se nas percepções dos gestores e funcionários da área de gestão ambiental as fontes referentes aos elementos da cultura de inovação, que orientam a adoção de práticas de ecoinovação. Essas fontes foram verificadas em cada uma das categorias de análise definidas no estudo. O quadro 2 apresenta cada categoria com suas respectivas fontes do ambiente comuns que favorecem a adoção de práticas de ecoinovação, relatadas pelos entrevistados. 
Quadro 2: Elementos da cultura de inovação e fontes do ambiente que favorecem a adoção de práticas de ecoinovação

\begin{tabular}{|c|c|}
\hline Categorias de Análise & $\begin{array}{l}\text { Fontes do ambiente comuns citadas pelos entrevistados, que favorecem } \\
\text { a adoção de práticas de ecoinovação }\end{array}$ \\
\hline Adaptabilidade e flexibilidade & Localidade da obra; Clientes; normas e certificações; e mercado. \\
\hline $\begin{array}{l}\text { Regulamentações e políticas } \\
\text { ambientais }\end{array}$ & $\begin{array}{l}\text { Adequação às regulamentações; Procedimentos ambientais diários; Práticas } \\
\text { exigidas pelas leis; e Projetos de execução das obras e normas ambientais. }\end{array}$ \\
\hline Orientação para o mercado & inovações tecnológicas; exigências do mercado; e tendências do mercado. \\
\hline Crenças e Valores & $\begin{array}{l}\text { Estratégias produtivas e operacionais; Missão; e Repasse dos valores } \\
\text { ambientais. }\end{array}$ \\
\hline Estrutura & $\begin{array}{l}\text { Diálogos, brigada e inovações; Quadros informativos e treinamentos; e } \\
\text { Tomada decisão. }\end{array}$ \\
\hline Estímulo à inovação & $\begin{array}{l}\text { Melhoria e novas ideias; Premiações e bônus; e Mudanças e caixa de } \\
\text { sugestões. }\end{array}$ \\
\hline Comunicação Aberta & $\begin{array}{l}\text { Comunicação dos procedimentos ambientais; Canais de comunicação; e } \\
\text { Comunicação verbal/direta. }\end{array}$ \\
\hline
\end{tabular}

Fonte: elaboração própria.

Percebeu-se que os indicadores do elemento "adaptabilidade e flexibilidade" incidem sobre a adoção de práticas de ecoinovação, considerando que evidenciam as abordagens flexíveis e adaptativas necessárias para as empresas atenderem às práticas ambientais e inovadoras, as quais mudam conforme as características das fontes do ambiente externo, como clientes e mercado. Dessa forma, a capacidade de adaptação permite solucionar demandas ou problemas advindos dos clientes e do mercado profissional, por meio de uma cultura capaz de ter comportamentos flexíveis. Nesse sentido, Hogan e Coote (2013) entendem que a abordagem flexível gera a criatividade na resolução de problemas e impulsiona a inovação. Esses comportamentos são obtidos quando a empresa tem como prática operacional a flexibilidade, que segundo Dombrowski et al. (2007), é um importante elemento da cultura organizacional, que proporciona inovação pela ênfase na orientação e no questionamento dos procedimentos. Dessa forma, uma cultura que tem como elemento a flexibilidade está mais apta a inovar, pois é uma cultura que incentiva a busca pela melhoria contínua e se adapta às mudanças que impactam no ambiente organizacional.

$\mathrm{Na}$ categoria "regulamentações e políticas ambientais" verificou-se que as práticas de ecoinovação das empresas da construção são definidas e orientadas com base em legislações, principalmente as exigências advindas de regulamentações, normas e leis. Estas, geralmente, são específicas do setor da construção, devido às suas características, como a alta geração de resíduos sólidos e o uso dos recursos naturais nos processos de operação. Também, a implementação dessas práticas é realizada principalmente a partir das diretrizes contidas no projeto de execução da obra e em um plano de gerenciamento de resíduos. Nesse contexto, a categoria "regulamentações e políticas ambientais" apresenta concepções trazidas por autores como Jabbour e Santos (2013) e Jerônimo (2014), os quais consideram que as organizações incorporam a adequação ao meio ambiente no desenvolvimento de suas atividades, para estarem em conformidade com as regulamentações, que abrangem as exigências legais.

$\mathrm{Na}$ categoria "orientação para o mercado" percebeu-se que as empresas da construção acompanham as tendências tecnológicas e inovadoras do mercado, relacionadas às práticas de ecoinovação. Esse acompanhamento é contínuo e realizado por meio de consultorias especializadas, pesquisas de mercado e participação em eventos como feiras e congressos. Assim, a existência do elemento orientação para o mercado na cultura de inovação das empresas proporciona atualização das suas atividades produtivas, conforme avanços tecnológicos relacionados à preservação do meio ambiente. Dessa forma, o mercado é visto como um fator que acarreta mudanças na cultura das organizações (FORTADO; FADIL, 2012) referentes à inovação e ao meio ambiente. 
Em “crenças e valores", a missão é uma fonte citada por quase todos os respondentes, porém, três deles entendem que o meio ambiente não é uma questão que precisa estar descrita na missão, mas uma consequência do setor de atuação e da execução dos processos e procedimentos das atividades produtivas e operacionais. Isso significa que as empresas coordenam seus negócios alinhados com as preocupações com o meio ambiente, ainda que com caráter competitivo, mas que faz com que elas cumpram seu papel referente à conscientização ambiental (JERÔNIMO, 2014; TO; TANG, 2014).

A categoria "estrutura" direciona e valoriza os aspectos do meio ambiente, como um elemento que requer a definição de características de cooperação e interação de grupo na tomada de decisão (MARTINS; TERBLANCHE, 2003). Assim, compreende-se que as decisões sobre o meio ambiente são tomadas de maneira conjunta, existindo o compartilhamento dessas decisões para todos os envolvidos na execução do projeto. Os processos de trabalho relacionados ao meio ambiente são transmitidos por meio de treinamentos de integração e avisos em murais. Essa estrutura pertence à camada mais visível da cultura denomina de artefatos, que é um nível de fácil identificação (SCHEIN, 2009; HOGAN; COOTE, 2013). Dessa forma, tanto a estrutura tangível de objetos materiais (cartazes, lixeiras e baias) como a intangível (diálogos), são vistas como elementos essenciais para o gerenciamento do conjunto de ações ambientais. Estas asseguram uma estrutura organizacional que valoriza a liberdade referente às propostas de práticas, que possibilitam a adoção de inovações ambientais, ainda que as principais decisões são tomadas pela cúpula das empresas. Esse contexto reforça o entendimento que os aspectos estruturais norteiam os processos de inovação nas empresas (SARAN; SERVIERI; KALLINY, 2009; GODOY; PEÇANHA, 2009).

O "estímulo à inovação" compreende os aspectos presentes na cultura e que são disseminados pela alta gerência e pelo grupo de trabalho, que incentivam a criatividade e a inovação. Esses contemplam o reconhecimento de novas ideias, a orientação para assumir riscos e experimentação, que propiciam a formalização de inovações com perspetivas ambientais (MARTINS; MARTINS; TERBLANCHE, 2004; MARTÍN-DE CASTRO et al., 2013; HOGAN; COOTE, 2013). Por meio dos dados coletados, percebeu-se que os estímulos presentes na cultura das empresas possibilitam aos trabalhadores buscarem soluções inovadoras.

Por meio dos dados coletados, percebeu-se que as empresas da construção têm alguns canais de "comunicação aberta" referentes ao meio ambiente que foram mais citados. Esses canais são o telefone, o e-mail e a internet. A comunicação direta/verbal é o principal meio de comunicação utilizado para transmitir os procedimentos ambientais aos colaboradores das obras. No entanto, algumas empresas apresentam canais de comunicação mais inovadores, ou seja, aqueles que surgiram mais recentemente, tais como WhatsApp ${ }^{\circledR}$ e Facebook ${ }^{\circledR}$. O elemento comunicação aberta, segundo Godoy e Peçanha (2009), é uma característica cultural das organizações inovadoras, que garante a disseminação de informações e conhecimentos, possibilitando a ocorrência dos processos de inovação, os quais podem ser orientados para atender às questões ambientais. Dessa forma, a existência desse elemento na cultura de inovação das empresas do setor da construção viabiliza a efetividade do fluxo de informações ambientais, contribuindo para a adoção de práticas de ecoinovação. Além disso, a compreensão clara das informações pode melhorar a qualidade dos resultados dessas práticas e proporcionar soluções alternativas que produzem inovação (HOGAN; COOTE, 2013), o que pode propiciar um diferencial de competitividade.

Com esses elementos da cultura de inovação e fontes do ambiente que favorecem a adoção de práticas de ecoinovação identificados, partiu-se para a definição dos indicadores. Esses indicadores são medidas que podem possibilitar que as empresas verifiquem os benefícios advindos das práticas de ecoinovação para a competitividade. Nesse contexto, Arundel e Kemp (2009) abordam que a mensuração da ecoinovação é relevante, considerando que a 
competitividade das empresas está gradualmente sendo atrelada à capacidade delas em adotar ecoinovações.

\subsection{INDICADORES PARA PRÁTICAS DE ECOINOVAÇÃO E PROPOSIÇÃO DE MODELO DE MENSURAÇÃO}

A incorporação de indicadores para adoção de práticas de ecoinovação responde às demandas requeridas pelo contexto de desafios socioambientais (ORGANIZAÇÃO PARA COOPERAÇÃO E DESENVOLVIMENTO ECONÔMICO, 2009) e econômicos, que podem ser explorados pelas empresas para obter competitividade. O Quadro 3 apresenta o modelo de mensuração, por meio da definição desses indicadores a partir dos elementos culturais, que são influenciados por fontes do ambiente externo e interno, bem como exemplifica as práticas de ecoinovação identificadas nas empresas analisadas, para obter capacidade competitiva.

Quadro 3: Modelo de Mensuração de Indicadores dos Elementos Culturais para Adoção de Práticas de Ecoinovação

\begin{tabular}{|c|c|c|c|c|}
\hline $\begin{array}{l}\text { Categoria de } \\
\text { análise }\end{array}$ & $\begin{array}{l}\text { Fontes do } \\
\text { Ambiente }\end{array}$ & $\begin{array}{l}\text { Indicadores para adoção de } \\
\text { práticas de ecoinovação }\end{array}$ & $\begin{array}{l}\text { Escala de } \\
\text { Notas }\end{array}$ & $\begin{array}{l}\text { Exemplos de práticas de ecoinovação } \\
\text { para obter capacidade competitiva }\end{array}$ \\
\hline \multirow{9}{*}{$\begin{array}{l}\text { Adaptabilidade e } \\
\text { Flexibilidade }\end{array}$} & \multirow{2}{*}{$\begin{array}{l}\text { Localidade da } \\
\text { obra }\end{array}$} & $\begin{array}{l}\text { Capacidade de adaptação e } \\
\text { flexibilização das práticas } \\
\text { ambientais. }\end{array}$ & & \multirow{2}{*}{$\begin{array}{l}\text { Proteção de nascentes e o } \\
\text { reflorestamento em conformidade com } \\
\text { o local da obra, o que propicia a } \\
\text { empresa estar apta para competir em } \\
\text { concorrências e para construir usinas } \\
\text { hidrelétricas. }\end{array}$} \\
\hline & & $\begin{array}{l}\text { Ações ambientais adequadas } \\
\text { devido ao local da obra. }\end{array}$ & & \\
\hline & \multirow{2}{*}{ Clientes } & $\begin{array}{l}\text { Flexibilidade na assimilação das } \\
\text { exigências ambientais dos } \\
\text { clientes. }\end{array}$ & & \multirow{2}{*}{$\begin{array}{l}\text { Patronizar como um atrativo para } \\
\text { conquistar o cliente e assim manter a } \\
\text { competitividade, a flexibilidade de } \\
\text { inovações ambientais, conforme o } \\
\text { padrão ambiental de cada cliente. }\end{array}$} \\
\hline & & $\begin{array}{l}\text { Adequação às especificidades } \\
\text { ambientais do projeto do } \\
\text { cliente. }\end{array}$ & & \\
\hline & \multirow[b]{2}{*}{$\begin{array}{l}\text { Normas e } \\
\text { certificações }\end{array}$} & $\begin{array}{l}\text { A flexibilização das práticas } \\
\text { ambientais são decorrentes das } \\
\text { certificações. }\end{array}$ & & \multirow{2}{*}{$\begin{array}{l}\text { Tratar a água usada para tirar o óleo das } \\
\text { máquinas, que com a adoção de novo } \\
\text { processo volta para o rio que abastece a } \\
\text { empresa, para se adequar as NBR's. } \\
\text { Pode afetar o sucesso competitivo da } \\
\text { empresa, pois para se manter no } \\
\text { mercado, ela precisa atender às } \\
\text { normativas. }\end{array}$} \\
\hline & & $\begin{array}{l}\text { Novos processos e serviços de } \\
\text { redução de impactos ambientais } \\
\text { nas obras a partir das NBR's e } \\
\text { NR's. }\end{array}$ & & \\
\hline & \multirow[b]{2}{*}{ Mercado } & $\begin{array}{l}\text { Investimentos na redução do } \\
\text { uso de materiais para adaptar-se } \\
\text { às demandas do mercado. }\end{array}$ & & \multirow{3}{*}{$\begin{array}{l}\text { Investimento em novas tecnologias para } \\
\text { a construção pré-fabricada, para } \\
\text { adaptar-se à condição do mercado de } \\
\text { obras mais econômicas e rápidas. Isso } \\
\text { reduz a geração de resíduos nas obras e } \\
\text { proporciona a abertura de um novo } \\
\text { negócio, o que favorece a } \\
\text { competitividade. }\end{array}$} \\
\hline & & \begin{tabular}{|l|} 
Adaptação às tecnologias do \\
mercado, para obter benefícios e \\
inovações ambientais.
\end{tabular} & & \\
\hline & \multicolumn{2}{|c|}{ Média da Categoria } & & \\
\hline \multirow{6}{*}{$\begin{array}{l}\text { Regulamentações } \\
\text { e políticas } \\
\text { ambientais }\end{array}$} & \multirow[b]{2}{*}{$\begin{array}{l}\text { Adequação a } \\
\text { Regulamentações }\end{array}$} & $\begin{array}{l}\text { Procedimentos ambientais } \\
\text { orientados por regulamentações. }\end{array}$ & & \multirow{2}{*}{$\begin{array}{l}\text { Alteração dos processos conforme } \\
\text { regulamentações sobre resíduos, para, a } \\
\text { partir das sobras de materiais, fazer dois } \\
\text { novos produtos, muretas e pedras } \\
\text { graduadas. Isso propicia um diferencial } \\
\text { competitivo. }\end{array}$} \\
\hline & & $\begin{array}{l}\text { Alterações de processos para } \\
\text { atender às regulamentações } \\
\text { ambientais. }\end{array}$ & & \\
\hline & \multirow{3}{*}{$\begin{array}{l}\text { Procedimentos } \\
\text { ambientais } \\
\text { diários }\end{array}$} & $\begin{array}{l}\text { Controles ambientais das } \\
\text { atividades diárias do canteiro. }\end{array}$ & & \multirow{3}{*}{$\begin{array}{l}\text { Adoção do sistema Sienge, que permite } \\
\text { melhorar o controle ambiental das } \\
\text { atividades diárias do canteiro, } \\
\text { possibilitando soluções para diminuir os } \\
\text { desperdícios nas obras. Essas soluções } \\
\text { podem melhorar o desempenho } \\
\text { organizacional para a competitividade. }\end{array}$} \\
\hline & & $\begin{array}{l}\text { Monitoramento dos } \\
\text { procedimentos de redução dos } \\
\text { impactos ambientais. }\end{array}$ & & \\
\hline & & $\begin{array}{l}\text { Cuidados com o meio ambiente } \\
\text { descritos em documentos. }\end{array}$ & & \\
\hline & $\begin{array}{l}\text { Práticas exigidas } \\
\text { pelas leis }\end{array}$ & $\begin{array}{l}\text { Cumprimento das leis } \\
\text { ambientais. }\end{array}$ & & $\begin{array}{l}\text { A partir do PGR, exigido por lei, } \\
\text { adotam-se práticas de reutilização de }\end{array}$ \\
\hline
\end{tabular}




\begin{tabular}{|c|c|c|c|c|}
\hline $\begin{array}{l}\text { Categoria de } \\
\text { análise }\end{array}$ & $\begin{array}{l}\text { Fontes do } \\
\text { Ambiente }\end{array}$ & $\begin{array}{l}\text { Indicadores para adoção de } \\
\text { práticas de ecoinovação }\end{array}$ & $\begin{array}{l}\text { Escala de } \\
\text { Notas }\end{array}$ & $\begin{array}{l}\text { Exemplos de práticas de ecoinovação } \\
\text { para obter capacidade competitiva }\end{array}$ \\
\hline & & $\begin{array}{l}\begin{array}{l}\text { Elaboração do Plano de Gestão } \\
\text { de Resíduos Sólidos (PGR). }\end{array} \\
\end{array}$ & & \multirow{2}{*}{$\begin{array}{l}\text { resíduos e sobras, como a construção de } \\
\text { novas formas para fazer dormentes da } \\
\text { sobra do concreto, reduzindo os custos, } \\
\text { o que pode contribuir para a } \\
\text { sobrevivência da empresa. }\end{array}$} \\
\hline & & $\begin{array}{l}\text { Adoção de práticas que não são } \\
\text { exigências legais. }\end{array}$ & & \\
\hline & \multirow{3}{*}{$\begin{array}{l}\text { Projetos de } \\
\text { execução das } \\
\text { obras e normas } \\
\text { ambientais }\end{array}$} & $\begin{array}{l}\text { Projeto de execução da } \\
\text { construção ambientalmente } \\
\text { responsável. }\end{array}$ & & \multirow{4}{*}{$\begin{array}{l}\text { Planejar o Projeto de execução da obra } \\
\text { conforme estratégias baseadas em } \\
\text { normas ambientais, visando obter } \\
\text { benefícios econômicos para a empresa, } \\
\text { como a redução dos gastos referentes à } \\
\text { recomposiçáo das áreas degradadas, o } \\
\text { que pode afetar a capacidade de a } \\
\text { empresa continuar competitiva no } \\
\text { cenário de atuação. }\end{array}$} \\
\hline & & $\begin{array}{l}\text { Benefício econômico obtido por } \\
\text { meio de normas ambientais. }\end{array}$ & & \\
\hline & & $\begin{array}{l}\text { Estratégias ambientais } \\
\text { conforme os parâmetros } \\
\text { normativos. }\end{array}$ & & \\
\hline & \multicolumn{2}{|c|}{ Média da Categoria } & & \\
\hline \multirow{10}{*}{$\begin{array}{l}\text { Orientação para } \\
\text { o Mercado }\end{array}$} & \multirow{3}{*}{\begin{tabular}{|l} 
Inovações \\
tecnológicas
\end{tabular}} & $\begin{array}{l}\text { Melhorias e inovações } \\
\text { realizadas com base em } \\
\text { consultorias e na participação } \\
\text { em feiras tecnológicas. }\end{array}$ & & \multirow{3}{*}{$\begin{array}{l}\text { Aplicar nas construções as inovações } \\
\text { tecnológicas do mercado para } \\
\text { prevenção do meio ambiente, tais como } \\
\text { sistema inovador de capacitação da } \\
\text { água pluvial, que pode ser um } \\
\text { diferencial em relação aos concorrentes, } \\
\text { proporcionando competitividade. }\end{array}$} \\
\hline & & $\begin{array}{l}\text { Pesquisa de mercado sobre } \\
\text { inovações ambientais. }\end{array}$ & & \\
\hline & & \begin{tabular}{|l|} 
Benefícios decorrentes de \\
inovações do mercado.
\end{tabular} & & \\
\hline & \multirow{3}{*}{$\begin{array}{l}\text { Exigências do } \\
\text { mercado }\end{array}$} & $\begin{array}{l}\text { Atendimento das condicionantes } \\
\text { ambientais. }\end{array}$ & & \multirow{3}{*}{$\begin{array}{l}\text { Cumprir exigências do mercado, como } \\
\text { um condicionante de poluição, } \\
\text { mudando o maquinário para gás; e } \\
\text { adotar um sistema de fabricação de laje, } \\
\text { que reduz o uso de materiais e o custo } \\
\text { da obra, pode levar o cliente a contratar } \\
\text { a empresa, o que é crucial para o } \\
\text { sucesso competitivo. }\end{array}$} \\
\hline & & $\begin{array}{l}\text { Modernização dos sistemas de } \\
\text { fabricação de acordo com } \\
\text { exigências ambientais. }\end{array}$ & & \\
\hline & & $\begin{array}{l}\text { Conquista de clientes devido } \\
\text { aos procedimentos ambientais. }\end{array}$ & & \\
\hline & \multirow{3}{*}{$\begin{array}{l}\text { Tendências do } \\
\text { mercado }\end{array}$} & $\begin{array}{l}\text { Decisões produtivas de acordo } \\
\text { com os aspectos ambientais do } \\
\text { mercado. }\end{array}$ & & \multirow{4}{*}{$\begin{array}{l}\text { Acompanhar as tendências ecológicas } \\
\text { do mercado e aplicá-las nos processos } \\
\text { produtivos, iniciando a fabricação de } \\
\text { novos produtos, como vigas e lajes } \\
\text { alveolares, que reduzem os resíduos nas } \\
\text { obras. Aplicar essas tendências pode } \\
\text { renovar as práticas produtivas da } \\
\text { empresa e, assim, mantê-la competitiva. }\end{array}$} \\
\hline & & $\begin{array}{l}\text { Procedimentos e equipamentos } \\
\text { conforme o que é atual e mais } \\
\text { ecológico. }\end{array}$ & & \\
\hline & & $\begin{array}{l}\text { Aplicar as tendências ecológicas } \\
\text { do mercado para ser } \\
\text { competitivo. }\end{array}$ & & \\
\hline & \multicolumn{2}{|c|}{ Média da Categoria } & & \\
\hline \multirow{8}{*}{ Crenças e Valores } & \multirow{3}{*}{$\begin{array}{l}\text { Estratégias } \\
\text { produtivas e } \\
\text { operacionais }\end{array}$} & $\begin{array}{l}\text { Implantação de valores e } \\
\text { crenças referentes ao meio } \\
\text { ambiente. }\end{array}$ & & \multirow{3}{*}{$\begin{array}{l}\text { Definir as estratégias produtivas e } \\
\text { operacionais com base nos valores e } \\
\text { crenças ambientais. A partir delas, optar } \\
\text { por práticas que reduzam o consumo de } \\
\text { água e a geração de resíduos. A } \\
\text { divulgação dessas práticas pode } \\
\text { favorecer a imagem da empresa e a } \\
\text { competitividade. }\end{array}$} \\
\hline & & $\begin{array}{l}\text { Estratégias produtivas e } \\
\text { operacionais direcionam as } \\
\text { práticas ambientais. }\end{array}$ & & \\
\hline & & $\begin{array}{l}\text { Reuniões com o objetivo de } \\
\text { melhorar aspectos ambientais. }\end{array}$ & & \\
\hline & \multirow{3}{*}{ Missão } & $\begin{array}{l}\text { A missão, a visão e os valores } \\
\text { apresentam propósitos } \\
\text { ambientais. }\end{array}$ & & \multirow{3}{*}{$\begin{array}{l}\text { Formalizar na missão, na visão e nos } \\
\text { valores princípios ambientais e de } \\
\text { inovação tecnológica, para conduzir as } \\
\text { atividades organizacionais. Esses } \\
\text { princípios podem orientar a empresa a } \\
\text { adotar tecnologias ecoinovadoras, como } \\
\text { o sistema de aquecimento solar para } \\
\text { piscina, criando, assim, um fator de } \\
\text { competitividade. }\end{array}$} \\
\hline & & $\begin{array}{l}\text { As atividades de produção } \\
\text { demandam crenças e valores } \\
\text { ambientais. }\end{array}$ & & \\
\hline & & $\begin{array}{l}\text { Os aspectos ambientais são } \\
\text { consequência do ramo de } \\
\text { atuação da empresa. }\end{array}$ & & \\
\hline & \multirow{2}{*}{$\begin{array}{l}\text { Repasse dos } \\
\text { valores } \\
\text { ambientais }\end{array}$} & $\begin{array}{l}\text { Os valores e crenças } \\
\text { relacionados ao meio ambiente } \\
\text { são repassados para os } \\
\text { colaboradores. }\end{array}$ & & \multirow{2}{*}{$\begin{array}{l}\text { Realizar eventos periódicos para } \\
\text { repassar aos colaboradores os valores } \\
\text { ambientais, pode conduzi-los a propor } \\
\text { ecoinovações incrementais, como } \\
\text { acoplar um beg impermeável no } \\
\text { compressor, para não contaminar o solo. }\end{array}$} \\
\hline & & $\begin{array}{l}\text { Eventos periódicos sobre os } \\
\text { valores ambientais para os }\end{array}$ & & \\
\hline
\end{tabular}




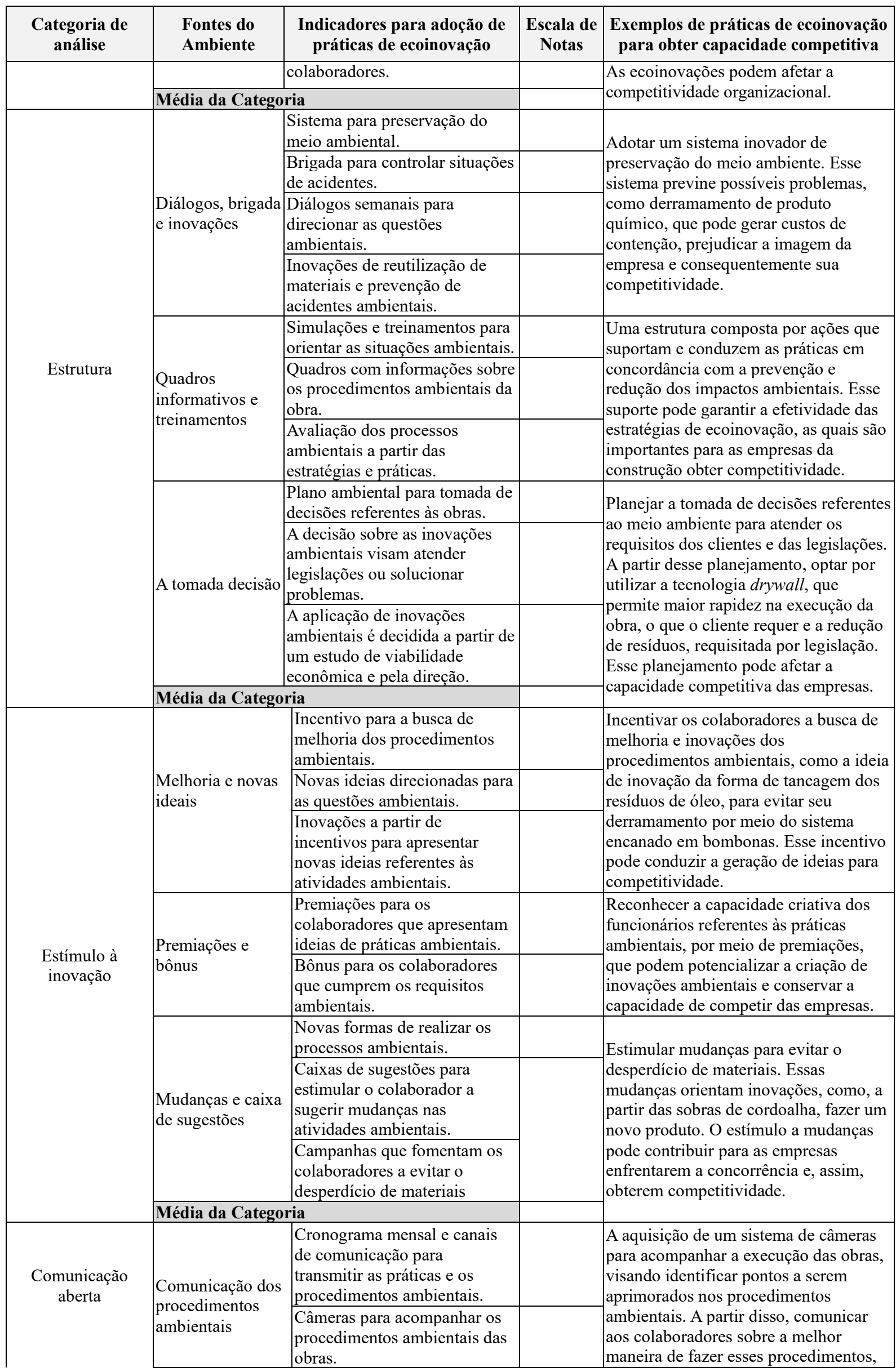




\begin{tabular}{|c|c|c|c|c|}
\hline $\begin{array}{l}\text { Categoria de } \\
\text { análise }\end{array}$ & $\begin{array}{l}\text { Fontes do } \\
\text { Ambiente }\end{array}$ & $\begin{array}{l}\text { Indicadores para adoção de } \\
\text { práticas de ecoinovação }\end{array}$ & $\begin{array}{l}\text { Escala de } \\
\text { Notas }\end{array}$ & $\begin{array}{l}\text { Exemplos de práticas de ecoinovação } \\
\text { para obter capacidade competitiva }\end{array}$ \\
\hline & & & & para conservar a competitividade. \\
\hline & \multirow{3}{*}{$\begin{array}{l}\text { Canais de } \\
\text { comunicação } \\
\text { (e-mail, telefone } \\
\text { e internet) }\end{array}$} & $\begin{array}{l}\text { Realização de melhorias nos } \\
\text { canais de comunicação da } \\
\text { empresa. }\end{array}$ & & \multirow{3}{*}{$\begin{array}{l}\text { Realização de melhorias nos canais de } \\
\text { comunicação da empresa, por meio do } \\
\text { investimento em mídias sociais, para } \\
\text { comunicar os processos referentes ao } \\
\text { meio ambiente aos colaboradores. } \\
\text { Quanto mais os colaboradores } \\
\text { conhecem os processos da empresa, } \\
\text { mais podem contribuir para o sucesso } \\
\text { deles, o que possibilita que as empresas } \\
\text { obtenham competitividade. }\end{array}$} \\
\hline & & $\begin{array}{l}\text { Mídias sociais para transmitir } \\
\text { as informações sobre processos } \\
\text { ambientais. }\end{array}$ & & \\
\hline & & \begin{tabular}{|l|} 
Utilização de diversos canais \\
para repassar os procedimentos \\
ambientais aos colaboradores
\end{tabular} & & \\
\hline & \multirow{3}{*}{$\begin{array}{l}\text { Comunicação } \\
\text { verbal/direta }\end{array}$} & $\begin{array}{l}\text { Um setor de referência para } \\
\text { transmitir as informações } \\
\text { ambientais. }\end{array}$ & & \multirow{4}{*}{$\begin{array}{l}\text { Realizar treinamentos sobre as técnicas } \\
\text { de comunicação verbal, visando sua } \\
\text { efetividade. Essa técnica é a mais usada } \\
\text { nas obras para o repasse das práticas } \\
\text { ambientais. Assim, a efetividade da } \\
\text { comunicação verbal possibilita que } \\
\text { essas práticas sejam realizadas } \\
\text { corretamente, demonstrando ao cliente a } \\
\text { competência da empresa, o que favorece } \\
\text { sua competitividade. }\end{array}$} \\
\hline & & $\begin{array}{l}\text { Treinamentos de técnicas de } \\
\text { comunicacão verbal. }\end{array}$ & & \\
\hline & & $\begin{array}{l}\text { Murais/quadros nas obras para } \\
\text { o repasse dos comunicados } \\
\text { relacionados ao meio } \\
\text { ambiente. }\end{array}$ & & \\
\hline & \multicolumn{2}{|c|}{ Média da Categoria } & & \\
\hline
\end{tabular}

Fonte: elaboração própria.

Para avaliar as fontes presentes nos elementos culturais que favorecem a adoção de ecoinovações, conforme a definição dos indicadores presentes no Quadro 3, sugere-se a utilização de uma escala de notas entre 0 (zero) à 10 (dez), contendo intervalos entre elas e parâmetros de análise, conforme níveis de execução. Essa escala e parâmetros são apresentados no Quadro 4.

Quadro 4: Escala e parâmetros de avaliação dos indicadores dos elementos culturais identificados
\begin{tabular}{|c|l|}
\hline $\begin{array}{c}\text { Escala de } \\
\text { Notas }\end{array}$ & \multicolumn{1}{c|}{ Parâmetros de Avaliação } \\
\hline 0 & $\begin{array}{l}\text { Não se executa, significa que não existe execução nas obras e/ou nos processos de produção da } \\
\text { empresa. }\end{array}$ \\
\hline 0,1 a 3,9 & $\begin{array}{l}\text { Pouca execução, significa que raramente tem execução nas obras e/ou nos processos de } \\
\text { produção da empresa. }\end{array}$ \\
\hline 4 a 6,9 & $\begin{array}{l}\text { Média execução, significa que regularmente tem execução nas obras e/ou nos processos de } \\
\text { produção da empresa. }\end{array}$ \\
\hline 7 a 9,9 & $\begin{array}{l}\text { Muita execução, significa que quase sempre tem execução nas obras e/ou nos processos de } \\
\text { produção da empresa. }\end{array}$ \\
\hline 10 & $\begin{array}{l}\text { Plena execução, significa que sempre tem execução nas obras e/ou nos processos de produção } \\
\text { da empresa. }\end{array}$ \\
\hline
\end{tabular}

Fonte: elaboração própria.

A partir das exposições dos Quadros 3 e 4, evidencia-se que os indicadores propostos no estudo podem servir para identificar as fontes do ambiente externo e interno presentes nos elementos da cultura de inovação, que conduzem ao desenvolvimento de práticas de ecoinovação. Essas práticas são adotadas principalmente para atender fontes do ambiente externo, como regulamentações ambientais e tendências ecológicas do mercado.

$\mathrm{O}$ atendimento das fontes externas possibilita a renovação das práticas produtivas, conforme normativas ambientais e inovações tecnológicas, o que, segundo Ansanelli (2011) e Silva (2013), podem afetar a capacidade de a empresa continuar competitiva no setor de atuação. Dessa forma, os resultados confirmam a percepção de Doran e Ryan (2012), de que as organizações podem potencializar a sua capacidade produtiva, por meio da adoção de 
ecoinovações, com o propósito de serem competitivas.

Para Ansanelli (2011), a melhoria de produtividade é orientada por regulamentações ambientais, que são vistas como uma fonte de oportunidades tecnológicas e competitivas. Nesse contexto, existe a necessidade de reestruturação nas formas de produção e consumo, conforme perspectivas ambientais que conduzem o processo de inovação. Este assume papel diferencial, tendo em vista que cada vez mais as novidades no âmbito organizacional impactam no posicionamento da empresa no mercado (Silva, 2013). Assim, para essa reestruturação acontecer, as empresas precisam desenvolver práticas de ecoinovação que se ajustem à competitividade organizacional.

\section{CONSIDERAÇÕES FINAIS}

Conforme evidenciado durante a pesquisa, os elementos da cultura de inovação podem contribuir para adoção das práticas de ecoinovação, pelo fato de direcionar os comportamentos dos membros da organização, orientando suas ações e práticas ambientais e inovadoras favoráveis à competitividade. Sob este enfoque, foram analisadas cinco indústrias do setor da construção, localizadas nas regiões Centro-Oeste e Oeste do Paraná. O objetivo foi de desenvolver um modelo de mensuração, por meio da definição de indicadores construídos a partir da análise dos elementos culturais, que possam favorecer a adoção de práticas de ecoinovação como fonte de capacidade competitiva.

Inicialmente, foram levantadas as fontes do ambiente referentes aos elementos da cultura de inovação, que orientam a adoção de práticas de ecoinovação. Essas fontes foram verificadas em sete categorias de análise dos elementos da cultura de inovação, para definir indicadores a partir dos pontos de congruências de cada categoria.

O primeiro elemento, "adaptação e flexibilização", apresentou as fontes de localidade da obra, clientes, normas e certificações, além do mercado. A partir desses fatores, as organizações do setor da construção precisam evidenciar uma abordagem flexível e adaptativa, necessária para que elas atendam às práticas ambientais e inovadoras, as quais mudam conforme as características das fontes do ambiente externo, como clientes e mercado. Assim, uma cultura adaptativa e flexível está mais apta a inovar, pois incentiva a busca pela melhoria contínua e se adapta às mudanças que impactam no ambiente organizacional.

Já no elemento "regulamentações e políticas ambientais" foram percebidas as fontes de: adequação às regulamentações; procedimentos ambientais diários; práticas exigidas pelas leis; e projetos de execução das obras e normas ambientais. Essas fontes favorecem a adoção de práticas de ecoinovação com o propósito de realizar o planejamento e o controle das práticas ambientais pertinentes à execução das obras, com base nas normas, leis e regulamentações, que se referem à geração e à destinação de resíduos das construções. Além disso, as empresas não definem de forma clara as políticas ambientais, mas têm seus procedimentos ambientais expressos geralmente em documentos como no projeto de execução da obra e no plano de gerenciamento de resíduos.

Sobre o elemento "orientação para o mercado" foram verificadas as fontes de: inovações tecnológicas; exigências do mercado; e tendências do mercado. Inferiu-se que as empresas acompanham as inovações tecnológicas, as exigências e as tendências do mercado relacionadas ao meio ambiente. Na cultura de inovação das empresas, o fator mercado tem grande importância, pois proporciona atualização das atividades produtivas, em face aos avanços tecnológicos relacionados à preservação do meio ambiente, principalmente pelo aspecto competitivo atual.

Quanto ao elemento "crenças e valores", foram definidas as fontes de: práticas produtivas e operacionais; missão; e repasse dos valores ambientais. No setor da construção, o meio ambiente não é visto como o cerne das crenças e valores presentes nas empresas, porém, é um 
fator desenvolvido em decorrência da execução dos procedimentos produtivos e operacionais das empresas.

O elemento "estrutura" na cultura de inovação nas empresas contem ações planejadas, tais como: diálogos, brigada e inovações; quadros informativos e treinamentos; e tomada de decisão. Neste caso, a estrutura é baseada em uma gestão que valoriza a liberdade referente às propostas de práticas de ecoinovação, entretanto, a decisão sobre a aplicação delas é centralizada geralmente na direção e nos clientes.

Constatou-se que o elemento "estímulo à inovação" fomenta a adoção de práticas por meio das fontes de: melhoria e novas ideais; premiações e bônus; e mudanças e caixa de sugestões, que incentivam o surgimento de novas formas de realizar as atividades ambientais. Nesse sentido, as empresas analisadas apresentaram uma cultura que incentiva o desenvolvimento da capacidade de propor ecoinovações dos colaboradores.

Na cultura de inovação das empresas do setor da construção, verificou-se que o elemento "comunicação aberta" apresenta como fontes: canais de comunicação; e comunicação verbal/direta. Esses canais são eficazes na disseminação das informações relacionadas ao meio ambiente, além de possibilitar a interação dos colaboradores que trabalham na sede da empresa com os pertencentes às obras. Além disso, percebeu-se que as empresas apresentam canais de comunicação comuns, como telefone, e-mail e internet, além de canais diferenciados em redes sociais, como WhatsApp ${ }^{\circledR}$ e Facebook ${ }^{\circledR}$.

A partir dessas fontes, foi definido um roteiro de diagnóstico de indicadores (conforme apresentado nos Quadros 3 e 4), a partir dos elementos da cultura de inovação, com base na identificação das fontes do ambiente externo e interno que orientam essas práticas. Este roteiro tem como propósito servir de fonte de informação e análise sobre os aspectos culturais que conduzem a adoção de ecoinovações. Além disso, possibilita identificar os avanços alcançados e a necessidade de melhorias e mudanças para obter resultados positivos referentes aos elementos da cultura de inovação, que favorecem a adoção de práticas de ecoinovação. $O$ estudo pode ser um mecanismo estratégico, uma vez que oferece um instrumento de avaliação, que propicia o feedback para as empresas referente ao desenvolvimento das práticas de ecoinovação.

Por fim, como limitação do estudo, aponta-se a questão geográfica, pelo fato de as empresas analisadas estarem localizadas nas regiões Centro-Oeste e Oeste do Paraná. Isso é decorrente da dificuldade de encontrar empresas disponíveis, de grande porte, para realização da pesquisa em outras regiões. Então, é importante considerar que o foco do estudo limitou o número de empresas em que a pesquisa pudesse ser realizada àquelas com porte maior, nas quais há mais possibilidade de existir práticas de ecoinovação. Além disso, ressalta-se que foram contatadas outras empresas, mas se recusaram a participar da pesquisa.

Assim, como sugestão de pesquisas futuras, recomenda-se aprofundar a relação dos elementos da cultura de inovação com as práticas de ecoinovação em outros segmentos, para ampliar o universo de pesquisas sobre os aspectos que favorecem a adoção de práticas de ecoinovação relacionadas aos elementos culturais. Também sugere-se aplicar os indicadores levantados em outras pesquisas do setor da construção, visando à obtenção de melhorias ambientais na construção dos futuros empreendimentos.

\section{AGRADECIMENTOS}

Agradecemos à Fundação Araucária de Apoio ao Desenvolvimento Científico e Tecnológico do Estado do Paraná (FA) pelo apoio à execução desta pesquisa. 


\section{REFERÊNCIAS}

ANSANELLI, S. L. M. Exigências ambientais europeias: novos desafios competitivos para o complexo eletrônico brasileiro. Revista Brasileira de Inovação, v. 10, n. 1, p. 129-60, 2011.

APEKEY, T. A.; MCSORLEY, G.; TILLING, M.; SIRIWARDENA, A. N. Room for improvement? Leadership, innovation culture and uptake of quality improvement methods in general practice. Journal of Evaluation in Clinical Practice, v. 17, n. 2, p. 311-8, 2011.

ARUNDEL, A.; KEMP, R. Measuring eco-innovation. [Working Paper Series]. UNU-MERIT. 2009. Disponível em: <http://www.merit.unu.edu/publications/wppdf/2009/wp2009-017.pdf $>$ Acesso em:10 abr. 2017.

BARDIN, L. Análise de conteúdo. 3. ed. Lisboa: Edições 70, 2004.

CARVAlHO, J. R. M. DE; CURI, W. W. F.; CARVAlHO, E. K. M. de A.; CURI, R. C. Proposta e validação de indicadores hidroambientais para bacias hidrográficas: estudo de caso na sub-bacia do alto curso do Rio Paraíba, PB. Revista Sociedade e Natureza, v. 23, n. 2, p. 294-310, 2011.

CIASULLO, M. V.; TROISI, O. Sustainable value creation in SMEs: a case study. The TQM Journal, v. 25, n. 1, p. 44-61, 2013.

DIAS, A. S. M. E. Proposta de um modelo de referência para a concepção e desenvolvimento de novos produtos. Tese (Doutorado em Engenharia e Gestão Industrial) Universidade da Beira do Interior, Covilhã, CB, Portugal, 2015.

DOMBROWSKI, C.; KIM, J.; SOUZA, K. de; BRAGANZA, A.; PAPAGARI, S.; BALOH, P.; JHA, S. Elements of Innovative Cultures. Knowledge and Process Management, Wiley InterScience, v. 14, n. 3, p. 190-202, 2007.

DORAN, J.; RYAN, G. Regulation and firm perception, eco-innovation and firm performance. European Journal of Innovation Management, v. 15, n. 4, p. 421-41, 2012.

FARIAS, A. S.; COSTA, D. S.; FREITAS, L. S.; CÂNDIDO, G. A. Utilização de eco-inovação no processo de manufatura de cerâmica vermelha. Revista de Administração e Inovação, v. 9, n. 3, p. 154-74, jul./set. 2012.

FORTADO, B.; FADIL, P. The four faces of organizational culture. Competitiveness Review: An International Business Journal, v. 22, n. 4, p. 283-98, 2012.

FRAJ, E.; MATUTE, J.; MELERO, I. Environmental strategies and organizational competitiveness in the hotel industry: the role of learning and innovation as determinants of environmental success. Tourism Management, v. 46, p. 30-42, 2015.

FREITAS, M. E. de. Cultura organizacional: grandes temas em debate. Revista de Administração de Empresas, v. 31, n. 3, p. 73-82, 1991.

GAZIULUSOY, I.; BREZET, H. Design for system innovations and transitions: a conceptual framework integrating insights from sustainability science and theories of system innovations and transitions. Journal of Cleaner Production, v. 108, p. 558-68, 2015. 
GODOI, C. K.; BALSINI, C. P. V. A pesquisa qualitativa nos estudos organizacionais brasileiros: uma análise bibliométrica. In: SILVA, A. B.; GODOI, C. K.; BANDEIRA-DEMELO, R. (Orgs.). Pesquisa qualitativa em estudos organizacionais: paradigmas, estratégias e métodos. São Paulo: Saraiva, 2010. p. 89-113.

GODOY, R. S. P.; PEÇANHA, D. L. Cultura organizacional e processos de inovação: um estudo psicossociológico em empresa de base tecnológica. Boletim. Academia Paulista de Psicologia, v. XXVI, n. 9, p. 142-63, 2009. Disponível em: $<$ http://www.redalyc.org/articulo.oa?id=94611474012> Acesso em: 18 mar. 2016.

GOMES, P. R.; MALHEIROS, T. F. Proposta de análise de indicadores ambientais para apoio na discussão da sustentabilidade. Revista Brasileira de Gestão e Desenvolvimento Regional, v. 8, n. 2, p. 151-69, 2012.

HOGAN, S. J.; COOTE, L. V. Organizational culture, innovation, and performance: a test of Schein's model. Journal of Business Research, v. 67, n. 8, p. 1609-21, 2013.

JABBOUR, C. J. C.; SANTOS, F. C. A. Empowerment dos funcionários e níveis de maturidade da gestão ambiental nas empresas: um modelo conceitual. Revista de Administração da UFSM, v. 6, n. 3, p. 497-510, set. 2013. Disponível em: $<$ http://www.redalyc.org/html/2734/273429771004/> Acesso em: 02 abr. 2016.

JANIUNAITE, B.; PETRAITE, M. The relationship between organizational innovative culture and knowledge sharing in organization: the case of technological innovation implementationin a telecommunication organization. Socialiniai Mokslai, v. 3, n. 69, p. 14-23, 2010.

JERÔNIMO, C. E. M. Diagnóstico da gestão ambiental em microempresas e empresas de pequeno porte da cidade de Manaus-AM. Revista Mestrado em Administração, v. 6, n. 1, p. 71-90, 2014.

KEMERICH, P. D.C.; RITTER, L. G.; BORBA, W. F. Indicadores de sustentabilidade ambiental: métodos e aplicações. Revista do Centro do Ciências Naturais e Exatas - UFSM, Revista Monografias Ambientais - REMOA, v. 13, n. 5, p. 3723-36, 2014.

KÖNNÖLÄ, T.; CARRILLO-HERMOSILLA, J.; GONZALEZ, P. del R. Dashboard of ecoinnovation. In: DIME International Conference - Innovation, sustainability and policy, 1113 September. University Montesquieu Bordeaux IV, France, 2008.

MAÇANEIRO, M. B.; CUNHA, S. K. da. Modelo teórico de análise da adoção de estratégias de ecoinovação reativas e proativas: a influência de fatores contextuais internos e externos às organizações. Brazilian Business Review, v. 11, n. 5, p. 1-24, 2014.

MACHADO, D. D. P. N. Inovação e cultura organizacional: um estudo dos elementos culturais que fazem parte de um ambiente inovador. Tese (doutorado em Administração) Escola de Administração de Empresas de São Paulo da Fundação Getúlio Vargas, São Paulo, 2004.

MARTÍN-DE CASTRO， G.; DELGADO-VERDE， M.; NAVAS-LÓPEZ， J. E.; CRUZGONZÁLEZ, J. The moderating role of innovation culture in the relationship between knowledge assets and product innovation. Technological Forecasting and Social Change, v. 80 , n. 2, p. 351-63, 2013. 
MARTINS, E.; MARTINS, N.; TERBLANCHE, F. An organizational culture model to stimulate creativity and innovation in a university library. Advances in Library Administration and Organization, v. 21, n. 4, p. 83-130, 2004.

MARTINS, E.; TERBLANCHE, F. Building organizational culture that stimulates creativity and Innovation. European Journal of Innovation Management, v. 6, n. 1, p. 64-74, 2003.

MEISSNER, J. O.; SPRENGER, M. Mixing method $s$ in innovation research: studying the process-culture-link in innovation management. Historical Social Research, v. 36, n. 1, p. 18098, 2011.

MELO, M. F. de S. de; YARYD, R. T.; SOUZA, R. C.; CAMPOS-SILVA, W. L. Responsabilidade social corporativa e competitividade: uma análise bibliométrica da evolução do tema. Revista Metropolitana de Sustentabilidade - RMS, v. 7, n. 2, p. 115-33, 2017.

NAGANO, M. S.; VICK, T. E.; MADEIRA, L. M. M. Suporte da gestão do conhecimento em práticas de ecoinovação. RISTI - Revista Ibérica de Sistemas e Teconologias de Informação, v. 22, p. 37-56, 2017.

NARANJO-VALENCIA, J. C.; JIMÉNEZ, D. J.; SANZ-VALLE, R. ¿Es la cultura organizativa un determinante de la innovación en la empresa? Cuadernos de Economía y Dirección de la Empresa, v. 15, n. 3, p. 63-72, 2012.

NARANJO-VALENCIA, J. C.; SANZ-VALLE, R.; JIMENEZ-JIMENEZ; D. Organizational culture as determinant of product innovation. European Journal of Innovation Management, v. 13, n. 4 , p. 466-80, 2010.

NILL, J.; KEMP, R. Evolutionary approaches for sustainable innovations policies: from niches to paradigm? Research Policy, v. 38, n. 4, p. 668-80, 2009.

ORGANIZAÇÃO PARA COOPERAÇÃO E DESENVOLVIMENTO ECONÔMICO, OCDE. Sustainable manufacturing and eco-innovation: framework, practices and measurement. Synthesis Report. France: OECD, 2009. Disponível em:

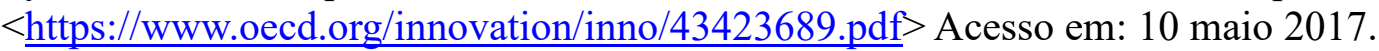

PACHECO-TORGAL, F.; LABRINCHA. J. A. The future of construction materials research and the seventh UN Millennium Development Goal: A few insights. Construction and Building Materials, v. 40, p. 729-37, 2013.

RENNINGS, K.; RAMMER, C. The impact of regulation-driven environmental innovation on innovation success and firm. Industry and Innovation, v. 18, n. 3, p. 255-83, 2011.

SANCHES, D. C. M.; GOMES, G. Cultura de inovação e sua influência na ecoinovação: estudo em empresas da indústria têxtil. Dissertação (Mestrado em Administração) Universidade Regional de Blumenau, SC, 2015.

SARAN, A.; SERVIERI, L.; KALLINY, M. Corporate culture, organizational dynamics and implementation of innovations: a conceptual framework. Asian Journal of Marketing, v. 2, n. 1, p. 10-9, 2009.

SCHEIN, E. H. Coming to a new awareness of organizational culture. Sloan Management Review, v. 25, n. 2, p. 3-16, 1984. 
SCHEIN, E. H. Cultura organizacional e liderança. São Paulo: Atlas, 2009.

SCHREIBER, D.; BESSI, V. G.; PUFFAL, D. P.; TONDOLO, V. A.G. Análise compreensiva da inovação em pesquisa \& desenvolvimento como prática cultural. Revista Perspectivas Contemporâneas, v. 11, n. 1, p. 55-80, 2016.

SCHUMPETER, J. A. Teoria do desenvolvimento econômico: uma investigação sobre lucros, capital, crédito, juro e o ciclo econômico. Tradutor: Maria Silvia Possas. São Paulo: Nova Cultural, 1997. (Os economistas).

SILVA, M. E. Inovação organizacional para o consumonsustentável: a inserção de novas práticas e tecnologiasnos negócios do Walmart Brasil. Reunir - Revista deAdministração, Ciências Contábeis e Sustentabilidade,v. 3, p. 66-89, 2013.

SINGH, N.; PARK Y. H.;TOLMIE, C. R.; BARTIKOWSKI, B. Green firm-specific advantages for enhancing environmental and economic performance. Global Business and Organizational Excellence. v. 34, n. 1, p. 6-17, 2014.

SOUZA, E. C. L.; LUCAS, C.C.; TORRES, C. V. Práticas sociais, cultura e inovação: três conceitos associados. Revista de Administração. FACES Journal. Belo Horizonte, v. 11, n. 2, p. 210-230, 2011. Disponível em: <file://C:/Users/Marize/Downloads/631-704-2PB\%20(1).pdf> Acesso em: 25 mar. 2017.

THEIS, V.; SCHREIBER, D. Inovação de produtos e processos sob a perspectiva da gestão ambiental. Revista de Ciências da Administração, v. 19, n. 47, p. 94-113, 2017.

TO, W. M.; TANG, M. N. F. The adoption of ISO 14001 environmental management systems in Macao SAR, China Trend, motivations, and perceived benefits. Management of Environmental Quality, v. 25, n. 2, p. 244-56, 2014. Disponível em: $<$ http://search.proquest.com/business/docview/1508379359/FEDA06A89E6C4B68PQ/2?acco untid=42153> Acesso em: 24 mar. 2017.

TURRO, Andreu; URBANO, David; PERIS-ORTIZ, Marta. Culture and innovation: the moderating effect of cultural values on corporate entrepreneurship. Technological Forecasting \& Social Change, v. 88, n. C, p. 360-9, 2014. 